\title{
Corela
}

Cognition, représentation, langage

3-2 | 2005

Vol. $3, n^{\circ} 2$

\section{La modification adjectivale en arabe à la lumière de la grammaire adaptative}

Adel Jebali

\section{CpenEdition}

\section{Journals}

Édition électronique

URL : http://journals.openedition.org/corela/574

DOI : $10.4000 /$ corela. 574

ISSN : $1638-573 \mathrm{X}$

\section{Éditeur}

Cercle linguistique du Centre et de I'Ouest - CerLICO

Référence électronique

Adel Jebali, «La modification adjectivale en arabe à la lumière de la grammaire adaptative », Corela [En ligne], 3-2 | 2005, mis en ligne le 27 décembre 2005, consulté le 01 mai 2019. URL : http:// journals.openedition.org/corela/574; DOI : 10.4000/corela.574

Ce document a été généré automatiquement le 1 mai 2019.

\section{(c) (i) (2)(2)}

Corela - cognition, représentation, langage est mis à disposition selon les termes de la licence Creative Commons Attribution - Pas d'Utilisation Commerciale - Partage dans les Mêmes Conditions 4.0 International. 


\title{
La modification adjectivale en arabe à la lumière de la grammaire adaptative
}

\author{
Adel Jebali
}

\section{Introduction}

1 Ce travail n'est qu'une ébauche d'une étude des aspects de la modification adjectivale en arabe dans le syntagme nominal. Une analyse plus poussée s'avérera nécessaire étant donné que ce phénomène est moins étudié dans la langue arabe que dans les langues indo-européennes, et surtout les langues romanes. On trouve, certes, des paragraphes çà et là dans les livres qui ont entrepris d'étudier la grammaire arabe, mais l'effort est presque exclusivement descriptif, voire normatif. Ainsi, les grammairiens arabes (à commencer par Sibawayhi au VIII ${ }^{\mathrm{e}}$ siècle) traitaient la modification adjectivale dans les chapitres consacrés à la catégorie Nom $^{1}$ ou dans les chapitres consacrés aux dérivés du verbe, voire même dans les chapitres consacrés aux dérivés en général. Ainsi, l'aspect syntaxique des adjectifs se trouve confondu avec l'aspect morphologique. Les orientalistes n'ont pas échappé à cette règle (Gaudefroy et Blachère: S.D.). Quant aux linguistes contemporains (par exemple Fassi Fehri 19932), rares sont leurs efforts consacrés à la modification adjectivale en arabe. Dans ce cadre, on peut considérer que l'exposé de Cantarino (1975) dans le chapitre voué à la modification des noms est un renouveau important dans l'étude de la question. Pourtant, il ne fournit que des éléments descriptifs dépourvus, dans la plupart des cas, d'explications.

Donc, étant donné la minceur des données bibliographiques pertinentes sur le sujet, ce travail va essayer de partir de la richesse des exemples présentés dans la tradition grammaticale et des intuitions de ces grammairiens pour tenter de poser un peu plus de questions, s'exercer à trouver des réponses, et parfois même des explications.

3 Le but n'étant pas seulement de décrire, mais aussi d'expliquer ${ }^{3}$, il s'agit dans ce travail de présenter une tentative d'explication qui a été proposée essentiellement pour le français 
et pour l'anglais et d'essayer de l'appliquer sur l'arabe. Une application qui vise essentiellement à poser des questions plus qu'à y répondre. Par conséquent, ne sont présentés ici que quelques éléments de réponse en vue d'émettre des hypothèses de travail et d'essayer de trouver des explications.

Cet article comporte trois parties. Dans la première, nous présenterons brièvement les solutions présentées dans Bouchard (2002) pour les problèmes que pose la modification adjectivale dans le syntagme nominal en français et en anglais. La seconde partie sera consacrée à une brève description de la modification adjectivale en arabe. Quant à la troisième partie, elle traitera des problèmes que pose l'application de la théorie adaptative à l'étude de cette langue. ${ }^{4}$

\section{La modification adjectivale en français et en anglais : l'approche de Bouchard}

Le contenu de cette partie est une présentation brève de la théorie et des hypothèses exposées dans Bouchard (2002). Aussi, ne seront exposées ici que les grandes lignes et ceci, d'une manière brève.

\subsection{Le problème de la compositionalité}

Bouchard a entrepris de résoudre un problème très ancien et très étudié dans la linguistique ainsi que dans d'autres domaines connexes (la logique par exemple). Il s'agit, en fait, du problème de la compositionalité de la modification adjectivale qu'on peut poser dans ces termes : quelques combinaisons d'adjectifs et de noms dans le cadre de la modification adjectivale semblent ne pas être compositionnelles (au sens de Frege); c'està-dire que le sens de l'expression résultant de la juxtaposition d'un adjectif et d'un nom dans un syntagme nominal n'est pas toujours fonction du sens de ses deux parties et de leur mode de combinaison.

Bouchard (2002 : chapitre 1) souligne trois propriétés de la modification adjectivale qui ne correspondent pas à nos attentes intuitives à propos de la compositionalité :

8 il y a beaucoup de cas où un SN fait à partir du même Adj et du même $\mathrm{N}$, avec apparemment la même relation syntaxique, donne le jour à une expression complexe avec plus qu'un seul sens. L'exemple typique est l'expression anglaise old friend qui peut signifier un ami âgé ou un ami de longue date.

La variation entre les langues: un certain sens exprimé par une combinaison syntaxique dans une langue est parfois exprimé par une combinaison syntaxique différente des mêmes parties équivalentes dans une autre langue. L'un des exemples qui illustrent ce cas serait homme pauvre et pauvre homme, qui équivaudraient à poor man en anglais.

10 Même si un $\mathrm{N}$ et un Adj expriment chacun une propriété qui définit un ensemble, la combinaison d'un Adj et d'un $\mathrm{N}$ n'est pas toujours interprétée comme une simple intersection d'ensembles. Les exemples que donne Bouchard sont les suivants: Carnivorous mammal, square table, red ball et married man.

11 Ces problèmes ont donné naissance à beaucoup d'études qui ont essayé de sauver la compositionalité moyennant des « engineering solutions » au sens de Chomsky (2001). Cet échec à fournir de vraies explications est surtout dû à la vision qu'avaient ces analyses de 
la syntaxe (les représentations syntaxiques sont étroitement basées sur ce qui est dérivé de l'ordre linéaire). Pour Bouchard, on peut avoir des explications si on change les hypothèses de travail en fondant la théorie sur l'ensemble complet des propriétés logiquement antérieures des interfaces de forme et de sens.

\subsection{La solution de Bouchard}

12 Considérant que tous les exemples de modification adjectivale qui paraissaient non compositionnels sont, par contre, compositionnels, Bouchard a proposé une explication basée sur des notions fondamentales de combinaison linéaire et de contribution sémantique, qui constituent des axiomes trouvant leur source dans les propriétés des systèmes externes logiquement antérieurs au langage (Bouchard 2002: conclusion du chapitre 2). On peut résumer cet effort en deux volets : le premier concerne la résolution des problèmes que pose la modification adjectivale en français et le deuxième, qui nous intéresse particulièrement ici, concerne l'application de la théorie qui en découle sur d'autres langues, notamment l'anglais (ainsi que les langues celtes, le wallon et le roumain, en second degré).

\subsubsection{La modification adjectivale en français}

13 Bouchard commence, tout d'abord, par définir le paramètre de la linéarisation pour le français, qui prend la forme suivante :

En français, la catégorie foncteur précède son dépendant.

14 Ainsi, l'ordre N+Adj est supposé être la forme la plus simple de l'association sémantique asymétrique dans laquelle le $\mathrm{N}$ est la tête et Adj est son dépendant. Pourtant, on trouve un autre ordre, tout aussi présent et productif dans cette langue, à savoir Adj+N. La différence entre les deux a été souvent pensée comme idiosyncrasique, ce qui n'est pas le cas, puisqu'on a aussi constaté des régularités qui régissent les deux formes. Pour Bouchard, la différence est dans la manière dont l'Adj se combine avec le N. En fait, dans la concaténation $\mathrm{N}+A d j$, l'Adj est en intersection avec tout le réseau sémantique de $\mathrm{N}^{5}$, alors que dans la concaténation Adj+N, il modifie un ou plus des sous composants internes à $\mathrm{N}$ ou dénote une propriété inhérente à $\mathrm{N}$.

15 En relation avec cette hypothèse démontrée par un grand nombre d'exemples, Bouchard a examiné d'autres concaténations possibles dans cette langue en profitant des propriétés des systèmes externes SM et CI pour expliquer les différences dans l'interprétation sémantique de ces formes. Ainsi, il a étudié les concaténations Adj + N propre, Adj1+Adj2 +N, Adj1+N+Adj2, N+Adj1+Adj2, les problèmes que pose l'emploi anaphorique des adjectifs prénominaux, les phénomènes de liaison et d'autres problèmes reliés aux facteurs qui favorisent l'emplacement prénominal ou postnominal des adjectifs.

16 Étant donné le caractère concis de cet article, nous nous contentons de cette présentation brève de la modification adjectivale en français du point de vue de Bouchard (2002). Nous mettrons plus l'accent sur l'application de cette théorie sur d'autres langues.

\subsubsection{La variation linguistique : le cas de l'anglais}

En anglais, comme en français, la tête précède son dépendant dans le cas «normal » où la relation encodée est entre une tête foncteur complet et un dépendant. Pourtant, l'anglais exclut généralement la concaténation $\mathrm{N}+$ Adj que le français permet ${ }^{6}$. Cette différence est 
due, selon Bouchard, à des choix différents effectués par ces deux langues parmi les moyens également valides de la réalisation du Nombre (désormais $\mathrm{Nb}$ ) que le système $\mathrm{SM}$ fournit. Le fait que le français encode le $\mathrm{Nb}$ sur le Déterminant (désormais Det) et que l'anglais l'encode sur le $\mathrm{N}$, a des effets sur la modification adjectivale. L'idée est que l'adjectif n'établit pas la même relation avec un $\mathrm{N}+\mathrm{Nb}$ qu'avec un $\mathrm{N}$ sans le $\mathrm{Nb}$ réalisé, parce que la sémantique de ces éléments n'est pas la même (cf. Bouchard 2002 : chapitre 3).

«We now see why the different ways in which French and English realize Number in a nominal plays a crucial role in the distribution of ADJs. In French, Number is on Det. Therefore, by an Elsewhere application of the Linearization Parameter, a bare Adj in prenominal position may relate to a subpart of the network of the $\mathrm{N}$ it combines with; conversely, a bare postnominal ADJ may relate to the whole network of the N. On the other hand, in English, Number is on the N. Therefore, a bare postnominal ADJ cannot relate to the $\mathrm{N}$ alone, but only to the atomized $\mathrm{N}$ +Number: So only a special kind of argument taking ADJ can appear in this position, because only such an ADJ has the semantics to appropriately modify an element with the semantics of an atomized N. Other "regular" bare ADJ must relate to the $\mathrm{N}$ alone. In order for such an ADJ to modify just the N, i.e., a subpart of the N+Number element, an Elsewhere application of the Linearization Parameter is required: the ADJ must appear in prenominal position. A similar Elsewhere application will also allow a prenominal "intentional" ADJ to access a subpart of $\mathrm{N}$, such as when the time interval indication is modified by a temporal ADJ" (Bouchard 2002: 175)

Mais comment, donc, l'anglais exprime-t-il les significations qu'exprime le français avec des Adj postnominaux? La réponse de Bouchard à cette question fait appel à l'intonation comme moyen utilisé par cette langue pour exprimer ces significations. L'intonation étant rigide en français, cette langue a recours à l'ordre de placement des Adj, alors que c'est un moyen productif en anglais qui se réalise de deux façons : l'accent contrastif et la pause (la virgule). Selon Bouchard, la langue a recours à ces moyens de codage quand la juxtaposition atteint une limite maximale d'expressivité (la notion de covariation fonctionnelle).

Il faut noter que Bouchard a essayé d'étendre cette analyse à d'autres langues (les langues celtes, le wallon et le roumain). Le présent travail est une tentative dans ce sens. Nous commencerons, tout d'abord, par fournir un cadre descriptif de la modification adjectivale en arabe et des problèmes qu'elle pose. Nous essaierons, ensuite, de voir si l'arabe se prête à une analyse telle que présentée dans la section 1 de ce travail (les chapitres 1 et 2 de Bouchard 2002).

\section{La modification adjectivale en arabe : une description}

Depuis Sibawayhi (VIII siècle) dans son livre "Al Kitaab", les grammairiens et linguistes qui ont traité l'aspect syntaxique et sémantique de la modification adjectivale en arabe ont partagé le consensus suivant : il y a deux sortes de qualification adjectivale en arabe : l'apposition adjectivale réelle, et l'apposition adjectivale dite sababii (connexe ou dérivée). 


\subsection{L'adjectif réel}

21 L'ordre de surface dans cette sorte d'adjectif est de la forme N+Adj. Cet ordre est censé être de base (dans le sens le plus large du terme) : l'adjectif vient modifier le nom qui le précède. Et, étant donné que le nom est le gouverneur ${ }^{7}$ de l'adjectif dans cette construction, l'accord entre les deux se réalise selon quatre traits :

- En genre (les genres possibles sont binaires : le masculin et le féminin) :
(1) a. rajul-u-n
homme-nom-un
kariim-u-n
'un homme généreux'
généreux-nom-un
b. ?imra?at-u-n kariima-t-u-n 'une femme généreuse' femme-nom-une généreux-fém-nom-une

- En nombre (singulier, duel et pluriel) :
(2) a. rajul-aa-ni baxil-aa-ni homme-nom-duel avare-nom-duel
b. rijaal-u-n buxalaa?-u
'des hommes avares'
hommes-nom-un avares-nom
'deux hommes avares'

- En Cas (nominatif, accusatif et génitif) :
(3) a. rajul-u-n
šujaa ${ }^{c}-u-n$
homme-nom-un courageux-nom-un
b. rajul-a-n
šujaa ${ }^{c}-a-n$
homme-acc-un courageux-acc-un
c. rajul-i-n
šujaac-i-n
homme-gén-un courageux-gén-un
'un homme courageux'
'un homme courageux'
'un homme courageux'

- En définitude (défini et indéfini) :
(4) a. bayt-u-n
jamiil-u-n
maison-nom-une
belle-nom-une
b. l-bayt-u l-jamiil-u
la-maison-nom la-belle-nom
'une belle maison'
'la belle maison'

Dans ces constructions, le foncteur est le $\mathrm{N}$ alors que le Adj joue le rôle du modifieur. Les tests syntaxiques pertinents nous démontrent que c'est l'adjectif qui s'accorde avec le nom et non pas l'inverse. Le paramètre de la tête en arabe a la forme suivante (voir Bouchard 2002:61):

(5) en arabe, la catégorie foncteur précède son dépendant. ${ }^{8}$

L'arabe a, donc, fait un choix entre les deux possibilités de linéarisations offertes par les propriétés de l'interface SM, un choix en faveur de l'ordre : foncteur+dépendant. Ce choix a un effet immédiat sur la modification adjectivale, mais aussi sur le rapport entre le verbe et ses arguments, et généralement sur la relation asymétrique entre la tête et son dépendant. Par conséquent, l'ordre $\mathrm{N}+\mathrm{Adj}$, en arabe, est l'ordre canonique, d'où l'affirmation suivante qu'on trouve dans presque tous les traités normatifs de la grammaire arabe «l'adjectif suit toujours le nom qu'il modifie et ne le précède jamais » (Hassan 1979).

Pourtant, il y a des cas où l'adjectif employé est suivi par un substantif (Cantarino, 1975 : 107) et apparaît donc dans une position non canonique, ce qui a des effets sur les phénomènes d'accord et sur la signification. (6) serait une illustration de ce cas :
(6) fii muxtalif-i
1-bilaad-i
'dans différents pays'
dans différent-gén les-pays-gén

«Arabic frequently uses an adjective followed by a substantival determination in the genitive case, where we would normally expect an adjectival apposition to the noun » (Cantarino, $1975: 107$ ) 
Ce phénomène est peu étudié en arabe, pour ne pas dire qu'il n'a jamais fait l'objet d'une étude détaillée. Cependant, il est clair que ce changement de l'ordre n'est pas fortuit et implique un changement sémantique. Cantarino est presque le seul linguiste à avoir essayé d'expliquer la différence sémantique que cette construction implique. En effet, l'ordre Adj+N exprimerait deux propriétés différentes, selon lui :

(a) L'adjectif exprime une idée abstraite : l'accord est affecté par cette signification de sorte qu'en (6), par exemple, l'adjectif est au singulier alors que le nom est au pluriel. On remarque aussi qu'il n'y a pas d'accord en définitude (le nom porte l'article défini et pas l'adjectif), ni en genre ni en Cas. En fait, dans ce genre de constructions adjectivales, l'adjectif est toujours au masculin singulier quels que soient les traits du nom modifié, d'où le contraste suivant :
(7) a. qadiim-u t-tawaariix-i 'les temps anciens' ancien-nom les-temps-gén

(b) L'adjectif exprime une idée concrète : dans ce cas, l'adjectif s'accorde avec le nom en genre et en nombre, mais pas en Cas ni en définitude, d'où (8) et (9) :
(8) Sighaar-u s-samak-i 'les petits [parmi] les poissons' petits-nom les-poissons-gén
(9) kabiir-aa-t-u l-xawaatiin-i 'les grandes [parmi] les femmes importantes' grand-pl-fém-nom les-femmes.importantes-gén

\begin{abstract}
Cantarino ne donne aucune explication à l'existence de telles constructions qui sont, apparemment, contradictoires avec la réalisation du paramètre de la tête en arabe. Il ne tire par ailleurs aucune conclusion quant aux phénomènes d'accord qui découlent de ce changement. Les grammairiens arabes de la tradition se contentent eux aussi de noter que dans ces constructions c'est l'adjectif qui gouverne le nom en lui assignant le Cas génitif. Ce qui est le plus surprenant c'est qu'aucun grammairien ou linguiste n'a traité les exemples en (6)-(9) comme illustrant des cas d'adjectifs prénominaux. Il y a unanimité sur le fait qu'il n'y a que des adjectifs postnominaux en arabe. ${ }^{9}$

29 Le problème mériterait beaucoup de réflexion et de recherche, d'autant plus que l'arabe reconnaît une deuxième sorte d'adjectifs qui suscite des interrogations.
\end{abstract}

\title{
2.2. L'adjectif dit sababii (ou connexe ${ }^{10}$ )
}

30 Cet adjectif ne porte pas sur le nom qu'il qualifie, mais sur un autre nom qui a un certain rapport avec celui-là (ou connexe à celui-là). Dans ce cas, l'adjectif, en tant que prédicat préfixé, porte sur le nom qui le suit, lequel est considéré comme étant son sujet, et les deux, ensemble, forment un qualificatif pour le nom qui précède l'adjectif (Wright 1898, V.2 : 283). On a, donc, une forme semblable à $\mathrm{N} 1+\mathrm{Adj}+\mathrm{N} 2$, où $\mathrm{N} 1$ gouverne le syntagme dont Adj est la tête et $\mathrm{N} 2$ un dépendant de cette tête.

En ce qui concerne l'interprétation sémantique de cette forme, peu d'explications ont été fournies. Pourtant, on trouve une bonne description dans Kouloughli (1994:113): «si on dit waladun jamiilun 'un bel enfant': litt. "enfant beau", on exprime que l'adjectif «beau» s'applique à l'enfant dans sa globalité. Si l'on veut restreindre la portée de 
l'adjectif et dire, par exemple, que la beauté se limite au visage de l'enfant, on dispose en arabe de deux constructions :

- La première consiste à ajouter à l'adjectif un complément spécificatif (...). Mais cette construction, très vivante pour les élatifs et dans d'autres parties de la grammaire, est devenue désuète en ce qui concerne les adjectifs réels.

- La seconde construction, très usitée, consiste à doter l'adjectif d'un complément de nom qui spécifie son domaine d'application. On dira donc waladun jamiilu lwajhi 'un enfant beau de visage' : litt. « enfant beau-[de]-le-visage » (fin de la citation).

Cette construction affecte l'accord d'une manière particulière. En fait, l'accord en définitude n'y est pas obligatoire, d'où la possibilité de (10)-(12) :

(10) huwa rajul-u-n Hasan-u l-wajh-i 'il est un homme au beau visage'

il homme-nom beau-nom le-visage-gén

(11) huwa r-rajul-u Hasan-u l-wajh-i 'il est l'homme au beau visage'

il le-homme-nom beau-nom le-visage-gén

(12) huwa r-rajul-u l-Hasan-u l-wajh-i 'il est l'homme au beau visage'

il le-homme-nom le-beau-nom le-visage-nom

L'accord en genre, en nombre et en Cas est, par contre, obligatoire.

Ces particularités d'accord révèlent un contraste (Cantarino 1975 : 109). En fait, l'adjectif connexe est en accord avec son nom gouverneur, mais, en tant que tête d'un syntagme, il réfère logiquement au génitif qu'il gouverne au sein de ce syntagme. En d'autres mots, N1 est en accord avec Adj, alors que sémantiquement parant, Adj modifie plutôt N2. En outre, l'accord entre Adj et N1 est limité; seuls les traits de genre et de nombre sont activés en plus du Cas morphologique :

(13) a. hiya ?imara?t-u-n Hasana-t-u l-wajh-i 'elle est une femme au beau visage' elle femme-nom-une beau-fém-nom le-visage-gén

b. huwa rajul-u-n Hasan-u l-wajh-i 'il est un homme au beau visage'

il homme-nom-un beau-nom le-visage-gén

(14) a. humaa rajul-aa-ni Hasan-aa l-wajh-i 'ils sont deux hommes au beau visage' ils.duel homme-nom-duel beau-nom.duel le-visage-gén

b. hum rijaal-u-n Hasan-u l-wajh-i 'ils sont des hommes au beau visage' ils hommes-nom-indéf beau-pl.sg le-visage-gén

Pourtant, cette construction comporte un certain accord entre Adj et N2 dans certains cas où l'adjectif sélectionne un sujet et lui assigne le Cas nominatif, agissant ainsi comme un verbe. Cependant, les propriétés d'accord qu'on trouve entre le verbe et le sujet postverbal ne sont pas les mêmes entre un adjectif et son gouverné (sujet). Le sujet postverbal ne s'accorde pas avec le verbe qui le gouverne en nombre, alors qu'il est, ainsi que le genre, un trait activé dans l'accord entre Adj et N2. (15) est supposé être sémantiquement équivalent à (16) :

(15) a.Hasun-a l-wajh-u 'le visage s'est embelli' s'embellit.3Pers.sg.masc le-visage-nom

b. Hasun-at l-wujuuh-u 'les visages se sont embellis' s'embellit-3Pers.sg.fém les-visages-nom

(16) a. rajul-u-n Hasan-u-n wajh-u-hu 'un homme dont le visage est beau' homme-nom-indéf beau-nom-indéf visage-nom-il

b. rijaal-u-n Hisaan-u-n wujuuh-u-hum 'des hommes dont les visages sont beaux'

hommes-nom-indéf beaux-nom-indéf visages-nom-ils

Il convient de noter les particularités du pronom affixé au N2 et est lié au N1 ${ }^{11}$ (voir la section 3.3), dans la construction avec Adj en (16a-b). Ce pronom est le lien d'accord le 
plus fort entre $\mathrm{N} 1$ et le syntagme qui forme son adjectif. On remarque dans ces constructions une tendance de Adj à réaliser un accord fort avec N2 plutôt qu'avec N1. En (17) par exemple, il n'y a aucun accord en genre ni en nombre entre Adj et N1, et seul le Cas morphologique réalise son accord.

(17) ?ayyaam-u-n šadiid-a-t-un Haraarat-u-haa 'de très chaudes journées' journées-nom-indéf tenace-nom-fém.sg-indéf chaleur-nom-elle arabe que des adjectifs postnominaux. Les exemples d'adjectifs connexes ne font que confirmer cette idée, puisque de toute façon le syntagme dont l'adjectif est la tête modifie le nom qui le précède, et c'est tout à fait normal d'avoir des phénomènes d'accord dans ce syntagme, en raison de la juxtaposition de la tête et de son dépendant.

(b) La deuxième hypothèse n'a jamais vu le jour dans la grammaire traditionnelle ni dans les quelques études linguistiques modernes et contemporaines de ce phénomène. Elle consiste à supposer l'existence d'adjectifs prénominaux en arabe. Loin d'être des cas isolés, ces adjectifs constituent des formes très productives, que ce soit en arabe classique ou en arabe moderne. Les exemples de (6) à (9) et de (10) à (17) seraient des illustrations de la forme Adj+N. Et ce n'est pas un hasard si l'arabe a eu recours à ces constructions pour exprimer les subtilités sémantiques de la modification adjectivale que la forme $\mathrm{N}$ +Adj ne peut exprimer.

Il convient d'étudier les conséquences de ces hypothèses et leurs implications sur l'architecture de la langue arabe en général. La première a connu un grand succès et était très fructueuse dans les études grammaticales, mais la deuxième n'a jamais été formulée auparavant à notre connaissance. Dans le paragraphe suivant, nous allons essayer de trancher la question en faveur de l'hypothèse (a).

\subsection{II n'y a que des adjectifs postnominaux}

41 Pourquoi les grammairiens de la tradition, ainsi que les grammairiens modernes, n'ont-ils reconnu qu'une seule sorte d'adjectifs? Là, les arguments ne sont pas toujours clairement formulés à cause de la prépondérance du descriptif et du normatif, mais on peut en formuler trois principaux :

42 Le premier argument est formulé par Sibawayhi : on peut omettre le nom et l'adjectif prendra sa place dans la construction (sa fonction grammaticale et son Cas). En outre, chaque fois qu'une construction permet cette omission, l'ordre de surface est toujours $\mathrm{N}$ +Adj et non pas Adj+N. Cela exclurait les formes Adj+N illustrées en (6), répétées ici en (18). En fait, l'omission du nom dans cette construction rend la phrase agrammaticale parce que l'adjectif est formellement annexé au nom (19).

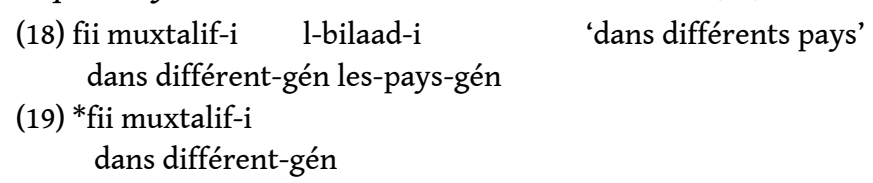

43 Le deuxième argument concerne les constructions avec l'adjectif connexe. En fait, la forme morphologique de l'adjectif (épithète) dans cette construction n'est pas toujours l'Adjectif (en tant que partie de discours) mais peut être aussi un participe actif. Dans ce cas, la relation entre Adj et $\mathrm{N} 2$ est altérée et ressemble plus à une relation entre un verbe et son argument interne. Il est difficile, ainsi, de parler d'apposition adjectivale :

Corela, 3-2 | 2005 
(20) rajul-u-n qaa?id-u-n jayš-a-hu 'un homme dirigeant son armée' homme-nom-indéf dirigeant-nom-indéf armée-acc-il la linéarisation et le paramètre du Nombre.

\subsubsection{Le paramètre de la linéarisation}

Il s'agit de définir l'ordre des constituants entre la tête et le dépendant. L'arabe a fait le même choix que le français et l'anglais en choisissant l'ordre: foncteur (tête) + dépendant. Cet ordre, qui a des effets sur tous les syntagmes, définit la relation entre le verbe et ses arguments, ainsi qu'entre les constituants de la phrase dite nominale (sujet / attribut). 


\subsubsection{Le paramètre du Nombre}

52 Tout comme l'anglais et les langues celtes, l'arabe a choisi de réaliser le $\mathrm{Nb}$ sur le $\mathrm{N}$. Néanmoins, la réalisation du $\mathrm{Nb}$ ne prend pas toujours la forme d'une désinence ou d'un suffixe sur le $\mathrm{N}$. $\mathrm{Nb}$ peut être exprimé par le schème (ou le thème) du N. L'exemple type de cette différence se trouve dans la distinction classique entre le pluriel «sain» ou externe et le pluriel «brisé » ou interne (Sibawayhi, Ibn Hichem, Gaudefroy et Blachère, et autres). Le pluriel externe équivaut à ce qu'on trouve en français, par exemple, et qui consiste à utiliser un suffixe pour distinguer le pluriel du singulier, sauf qu'en arabe, le suffixe est fléchi selon le genre et le Cas du N. Ainsi, il y des suffixes pour le masculin qui sont fléchis selon le Cas comme le montre le tableau en (23) :

(23) la flexion des désinences du pluriel externe masculin selon le Cas

\begin{tabular}{|l|l|l|l|}
\hline Le Cas & Le nominatif & L'accusatif & Le génitif \\
\hline \hline Le suffixe & uuna & iina & iina \\
\hline
\end{tabular}

Les suffixes du féminin sont eux aussi fléchis selon le Cas comme le montre le tableau en (24) :

(24) la flexion des désinences du pluriel externe féminin selon le Cas

\begin{tabular}{|l|l|l|l|}
\hline Le Cas & Le nominatif & L'accusatif & Le génitif \\
\hline \hline Le suffixe & aatu & aati & aati \\
\hline
\end{tabular}

54 Le pluriel «sain» est d'un emploi restreint en arabe ${ }^{14}$. Le pluriel le plus fréquemment utilisé n'a aucune désinence, est de même racine que le nom pris pour singulier, mais est construit sur un autre thème (ou schème). Le thème du pluriel est différent de celui du singulier par la nature ou la valeur de ses voyelles, longues ou brèves, et par des préfixes et suffixes, d'où la qualification de ce pluriel de «brisé » ou interne.

55 La description du Nombre se complique, en arabe, par l'ajout d'une autre valeur, à savoir le duel qui, même s'il tend à disparaitre dans les dialectes, reste très productif en arabe standard.

56 L'idée que l'arabe code $\mathrm{Nb}$ sur $\mathrm{N}$ plutôt que sur Det est démontrée par plusieurs tests syntaxiques. Nous pourrons aussi procéder par élimination en commençant par examiner l'hypothèse selon laquelle $\mathrm{Nb}$ est codé sur Det. Rien, en arabe, ne pourrait appuyer cette hypothèse. En fait, les articles défini et indéfini ${ }^{15}$ ne portent aucun trait et restent aveugles quant aux traits de $\mathrm{N}(\mathrm{Nb}, \mathrm{Gr}$, Pers, etc.) : «The indefinite, as well as the definite article, is unchangeable in gender, number, and Case; it occurs in singular only" (Kramsky: 1972).

(25) l-walad-u (l'enfant), l-walad-aani (les deux enfants), 1-?awlaad-u (les enfants)

(26) walad-u-n (un enfant), walad-aani (deux enfants), ?awlaad-u-n (des enfants)

57 Le deuxième choix, que ferait l'arabe parmi ce que les propriétés de l'interface SM lui fournissent, serait d'encoder le $\mathrm{Nb}$ sur un mot indépendant (comme le supposent quelques études du wallon). Il est clair que ce n'est pas le cas pour l'arabe, étant donné 
l'analyse que nous venons de faire de la réalisation $\mathrm{du} \mathrm{Nb}$ dans l'expression nominale. Il reste, donc, une seule possibilité logique : le Nb est encodé sur le N. Notons ici que ce ne serait pas possible à l'arabe d'encoder $\mathrm{Nb}$ sur Adj, pour la simple raison qu'Adj n'est pas obligatoire dans l'expression nominale. Pourtant, le Nb est visible sur $\mathrm{N}$ et Adj dans la plupart des cas, mais un système économique devrait encoder $\mathrm{Nb}$ une seule fois dans l'expression nominale pour des raisons d'interprétation sémantique. Ceci exclut la possibilité d'une double réalisation de $\mathrm{Nb}$ sur $\mathrm{N}$ et Adj.

\subsection{Le problème pour la grammaire adaptative}

L'analyse de Bouchard prévoit une corrélation entre la réalisation du Nb et la position des Adj, de sorte qu'une langue qui encode le $\mathrm{Nb}$ sur $\mathrm{N}$ (comme l'anglais) favoriserait la position prénominale alors qu'une langue qui l'encode sur le Det (comme le français) aura la possibilité des deux positions prénominale et postnominale. Cette corrélation est expliquée par des propriétés logiquement antérieures au langage et prédit des phénomènes qui en découlent directement. Ainsi, les langues qui encodent le $\mathrm{Nb}$ sur $\mathrm{N}$ permettent l'omission du Det et mettent des conditions strictes sur l'omission du N. Par contre, les langues qui encodent le $\mathrm{Nb}$ sur le Det, permettent une plus grande flexibilité quant à l'omission du N, mais sont plus contraignantes quand il s'agit de l'omission du Det. Qu'en est-il de l'arabe?

59 L'arabe encode le $\mathrm{Nb}$ sur le $\mathrm{N}$ tout comme l'anglais, pourtant il permet une seule position canonique pour les Adj «nus» ainsi pour les adjectifs avec compléments, à savoir la position postnominale. En plus, il permet l'omission du N, mais il ne permet pas l'omission du Det. Une expression nominale doit toujours avoir un Det (si ce n'est pas un article, alors un pronom démonstratif ou un $\mathrm{N}$ - lexical ou pronominal - annexé). Il se comporte, donc, comme le français en ce qui concerne la possibilité de l'omission du $\mathrm{N}$ et la restriction (voir l'impossibilité) de l'omission du Det, mais il se comporte comme l'anglais en ce qui concerne l'impossibilité de clitiques issus de la classe des Det ${ }^{16}$.

60 Ce cas met la généralisation de Bouchard devant un défi sérieux et demanderait un affinement de l'analyse qui dépasserait le cadre de ce travail. Cependant, on peut essayer d'apporter quelques éléments de réponse en vue d'une étude future plus poussée.

\subsection{Une solution?}

61 Le présent travail n'est qu'une ébauche, aussi il se contente de questionner et de poser des problèmes. Contre toute apparence, cette approche n'est pas étrangère à la science moderne, étant donné une certaine définition de la falsification ${ }^{17}$. Ainsi, nous avons essayé de définir le plus clairement possible les problèmes que rencontre l'analyse adoptée par Bouchard de la modification adjectivale quand il s'agit de prendre en considération la langue arabe. Il est clair qu'il est difficile de concilier certaines des propriétés de cette langue avec l'analyse adoptée et que certaines de ces propriétés vont à l'encontre même de ce que prédit la théorie adaptative. Si le Nombre joue un rôle dans la distribution des adjectifs en français et en anglais, il ne joue peut-être pas le même rôle en arabe. Pour ne donner qu'un exemple, l'adjectif, s'il est réalisé, ne s'accorde pas toujours avec le $\mathrm{N}$ en $\mathrm{Nb}$, d'où la possibilité de (27) où le $\mathrm{N}$ est omis :

(27) a. ra?ay-tu l-mar?at-a l-kuramaa?-a ?ajdaad-u-haa vu-je la-femme-acc les-généreux-acc ancêtres-nom-elle 
'j'ai vu la femme dont les ancêtres sont généreux'

b. ra?ay-tu l-kuramaa?-a ?ajdaad-u-haa

vu-je les-généreux-acc ancêtres-nom-elle

'j'ai vu [celle dont] les ancêtres sont généreux'

Dans ce cas, la tête du syntagme qui réalise l'adjectif porte un $\mathrm{Nb}$ sémantiquement et syntaxiquement différent de celui que devrait avoir le $\mathrm{N}$ s'il n'était pas omis. Le seul moyen de récupérer le $\mathrm{Nb}$ de $\mathrm{N}$ est le pronom haa ( $3^{\mathrm{e}}$ Pers., Sing., Fém.) affixé au sujet du syntagme qui fonctionne comme un adjectif. Il faut remarquer que sans ce pronom, la phrase serait agrammaticale. Est-ce que cela signifie que ce pronom serait le porteur sémantique du $\mathrm{Nb}$ dans l'expression nominale avec adjectif?

Les grammairiens de la tradition ont développé un point de vue selon lequel il y a toujours un pronom apparent ou "voilé » dans l'adjectif. On pourrait donc dire que ce pronom est un porteur potentiel du Nb sémantique. Mais si l'adjectif n'est pas un élément obligatoire de la phrase, on ne peut pas soutenir cette idée, d'autant plus qu'elle stipule un élément pronominal abstrait pour expliquer les phénomènes d'accord entre $\mathrm{N}$ et $\mathrm{Adj}$ même là où ce n'est pas nécessaire comme en (28) :

(28) r-rabiic $-u$ faSl-u-n jamiil-u-n 'le printemps est une belle saison' le-printemsp-nom saison-nom-indéf belle-nom-indéf

Nous pensons que nous n'avons pas besoin de ce pronom pour expliquer l'accord entre $\mathrm{N}$ et Adj et que c'est une opération coûteuse pour la théorie que de stipuler son existence. Mais, s'il y a des arguments assez forts qui prouveraient que l'intuition des grammairiens était fondée, ce qui reste à faire, l'élément pronominal stipulé (et réalisé dans le cas de l'adjectif connexe) fournirait une amorce d'explication. On pourrait ainsi dire que le $\mathrm{N}$ en arabe ne se combine pas avec Adj, mais que $\mathrm{N}+\mathrm{Nb}$ se combinent avec Adj+Pronom, et que cette configuration ne permet pas à l'adjectif d'être prénominal parce que le pronom ne précède jamais son antécédent en arabe. Mais cela signifie que ce n'est pas le Nombre qui a une incidence sur la distribution des adjectifs dans cette langue, mais plutôt les propriétés de liage (binding) des $\mathrm{N}$ et des pronoms.

65 Le comportement des adjectifs en arabe soulève d'autres problèmes. Prenons par exemple le Cas. L'arabe est une langue à flexions pour le Cas et distribue les éléments de la phrase selon ce critère. Aussi, le Cas joue un rôle très important dans la modification adjectivale. En fait, il est le seul trait d'accord entre $\mathrm{N}$ et Adj qui n'est jamais neutralisé et qui est obligatoirement réalisé dans l'expression nominale. C'est pourquoi quand le $\mathrm{N}$ est omis, le Cas doit être porté par Adj. C'est aussi le Cas qui permet la réalisation des phrases telles que (29) où le complément de l'adjectif est placé entre $\mathrm{N}$ et Adj, ce qui serait agrammatical en français (30):

(29) rajul-u-n bi-?ibnat-i-hi faxuur-u-n 'un homme fier de sa fille' homme-nom-indéf de-fille-gén-lui fier-nom-indéf

(30) *un homme de sa fille fier.

L'arabe ne se contente pas d'utiliser la juxtaposition entre $\mathrm{N}$ et Adj pour exprimer la relation de modification, c'est pourquoi plusieurs éléments peuvent s'interposer entre les deux. La réalisation du Cas permet cette flexibilité. Le français ne le permet pas, étant donné que les désinences casuelles ne sont pas visibles. Le français a donc besoin de la juxtaposition pour exprimer la relation sémantique entre $\mathrm{N}$ et Adj, mais l'arabe peut s'en passer. L'idée est que le Cas y est pour quelque chose dans la distribution des adjectifs et contribue, certes, à leur interprétation. Par conséquent, il faut en tenir compte si on entreprend d'émettre des hypothèses dans ce domaine. 

(Sibawayhi, Zamaxšarii, Ibn Hichem, Wright 1896 etc.) ont observé que l'origine de l'article défini est un démonstratif: "though it has become determinative, it was originally demonstrative " (Wright, V.1, 269). Leur argument principal, ce sont les cas où l'emploi de cet article apparait comme un démonstratif, surtout dans les mots l-yawm$a$ litt. ce jour (aujourd'hui) et $l$-?aan- $a$ litt. ce moment (maintenant). Le fait que ce même article apparaît dans plusieurs pronoms démonstratifs fournit un appui pour cette idée. Est-ce la preuve que l'article défini portait le $\mathrm{Nb}$ à certains stades de l'évolution de la langue arabe? Les démonstratifs sont, certes, porteurs d'une marque de Nombre, mais l'article défini, même dans ces emplois démonstratifs, peu productifs après tout, ne porte aucune marque de ce genre. Cela est dû, à notre avis, au statut particulier du Det en arabe, qui ne sert qu'à des fins de définitude. L'article défini constitue un moyen parmi trois de déterminer un $\mathrm{N}$. Les deux autres étant l'annexion d'un $\mathrm{N}$ et l'annexion d'un pronom clitique (affixé à $\mathrm{N}$ ). ${ }^{18}$

68 Le Det, en arabe, a donc des traits spécifiques et il est peut-être utile de prendre cette spécificité en considération pour avoir une vue d'ensemble de la modification de $\mathrm{N}$ dans cette langue. Nous pensons que ce statut particulier ne nous permet pas de considérer le Det comme la tête de l'expression nominale, comme font les analyses qui stipulent l'existence de la catégorie fonctionnelle DP (le syntagme déterminant ou SD). Il est clair que la projection de $\mathrm{N}$ est un $\mathrm{N}$ dans ce cas, étant donné la «nudité » du Det de toute marque de Nombre ou de Genre.

exposés ici font partie d'un plus grand ensemble de données dont il faut tenir compte pour étudier la modification adjectivale en arabe. Le caractère concis de ce travail ne nous permet pas d'aller plus loin dans l'investigation de cette question qui constitue un projet de recherche à part entière.

\section{Conclusion}

70 Nous avons essayé, dans ce travail, de fournir quelques éléments de réponse à quelquesuns des problèmes que pose la modification adjectivale au sein du SN en arabe. Le but était d'appliquer la théorie de la grammaire adaptative proposée dans Bouchard (2002). Aussi, nous avons tout d'abord exposé les grandes lignes de cette théorie dans le domaine de la modification adjectivale. Nous avons, par la suite, essayé d'apporter une description du comportement de la modification adjectivale en arabe en traitant, notamment, la question de la position des Adj dans cette langue. La position postnominale étant, pour la plupart des grammairiens et linguistes, la favorite, nous avons cherché à trouver une explication pour ce consensus. Nous avons, finalement, proposé une application des hypothèses de Bouchard (2002) sur l'arabe en révélant d'autres problèmes et d'autres données dont il faut tenir compte dans une étude plus détaillée.

71 La modification adjectivale en arabe n'était le sujet d'aucune recherche linguistique détaillée et exigerait plus d'efforts pour délimiter ces propriétés. Notre travail n'est qu'un essai dans ce sens, mais il reste incomplet du fait de son caractère bref. Aussi faut-il revenir sur la question pour apporter d'autres éléments de réponse. Les solutions proposées dans Bouchard (2002) sont de ce point de vue extrêmement précieuses parce qu'elles fournissent un modèle complet d'explication qui trouve sa source dans les 
propriétés logiquement antérieures des interfaces et non pas dans des stipulations qui reformulent, dans la plupart des cas, la description des phénomènes.

\section{BIBLIOGRAPHIE}

Bouchard D. 2002. Adjectives, Number and Interfaces: why languages vary. North Holland, Elsevier.

Boudelaa S. \& Gareth Gskell M. 2002. A Re-examination of the Default System for Arabic Plurals. In: Language and Cognitive Processes, 17(3), 321-343.

Cantarino V. 1975. Syntax of Modern Arabic Prose. Indiana University press, Bloomington/London.

Chomsky N. 2001. Beyond Explanatory Adequacy. MIT Occasional Papers in Linguistics. 20, 1-28. MIT Linguistics Department, Cambridge.

Gaudefroy M. \& Blachère R. (S.D.). Grammaire de l'arabe classique. Librairie Orientale Américaine.

Hasan A. 1987. En NaHw El Waafii.Le Caire, Daar El macaarif.

Ibn Hichem (X ${ }^{\mathrm{e}}$ siècle). Mughnil-labiib. URL : www.alwaraq.com

Jebali A. 1998. Des problèmes méthodologiques dans la linguistique moderne à la lumière du livre de J.C. Milner (1989) Introdution à une science du langage. Dissertation présentée comme exigence partielle pour obtenir le D.E.A. Université de Tunis I. Faculté des lettres de la Manouba.

Jebali, A. 2005. Les pronoms clitiques nominatifs du perfectif en arabe standard : vers une analyse lexicaliste. À paraître in Toronto Working Papers in Linguistics. Conférence donnée au Congrès annuel de Niagara Linguistic Society.

Kouloughli D.E 1994. Grammaire de l'arabe d'aujourd'hui. Pocket, Langues pour Tous.

Kramsky J. 1972. The Article and the Concept of Definiteness in Language. Mouton, Paris/The Hague.

Laenzlinger C. 2000. French Adjective Ordering: Perspectives on D-P Internal Movement Types.

Generative Grammar in Geneva, $1: 55-104$.

Lakatos I. 1970. Falsification and the Methodology of Scientific Research Programmes. In: Criticism and the Growth of Knowledge (Imre Lakatosand Alan Musgrave, eds.), 91-196. Cambridge University Press, Cambridge.

Milner J.C. 1989. Introdution à une science du langage. Seuil, Paris.

Sibawayhi (VIIIe siècle). El Kitaab. URL : www.alwaraq.com

Wright W. 1896. A Grammar of the Arabic Language. Cambridge University Press.

Zamaxšarii J.E. (X $\mathrm{X}^{\mathrm{e}}$ siècle). El MufaSSal. URL : www.alwaraq.com 


\section{NOTES}

1. À noter qu'ils considéraient les Adjectifs comme faisant partie de cette catégorie. Nous utilisons ici le terme Nom en tenant compte d'un autre terme aussi souvent utilisé pour dénoter cette catégorie; à savoir le Substantif.

2. Fassi Fehri a consacré un livre pour essayer d'appliquer la théorie du gouvernement et du liage à la langue arabe (1993). Il a étudié les adjectifs au chapitre 4 consacré aux catégories temporelles, aspectuelles et modales. Les problèmes qu'il a traités sont peu révélant pour ce sujet, étant donné qu'il a surtout mis l'accent sur les propriétés morphologiques, catégorielles et fonctionnelles des adjectifs et des participes. Les propriétés sémantiques et syntaxiques de la modification adjectivale sont mises de côté.

3. Une adéquation explicative qui n'est pas forcément pensée à la chomskyenne. En fait, il ne s'agit pas d'essayer de ramener des phénomènes inexplicables à des propriétés de la Grammaire Universelle, mais plutôt d'essayer de trouver des corrélations entre ces phénomènes, d'une part, et d'autres phénomènes dans la langue étudiée ainsi que les propriétés des interfaces SM et $\mathrm{CI}$ (Bouchard, 2002), d'autre part.

4. Ce travail n'est qu'une esquisse d'une étude plus détaillée de la question; il ne prétend pas cerner toutes les propriétés de la modification adjectivale en arabe, ni toutes les possibilités que fournissent les hypothèses de Bouchard.

5. Un réseau composé des éléments suivants: la fonction caractérisante $f$, la fonction d'assignation variable $g$, l'intervalle de temps $i$, et le monde possible $w$.

6. Il faut noter qu'il y a des cas en anglais où l'adjectif se réalise dans la position postnominale et qu'en français, certains nominaux ne prennent jamais d'adjectifs prénominaux.

7. Nous utilisons le mot gouverneur au sens que lui donnaient les grammairiens arabes classiques et non au sens technique qu'il a eu dans la grammaire générative. Ainsi, le gouverneur est, dans la théorie grammaticale traditionnelle, un élément de la phrase qui assigne un cas à un autre élément. Selon cette théorie, il y deux sortes de gouverneurs :

Les gouverneurs linguistiques manifestes ou perceptibles : hiérarchisés comme suit: V(erbe) $>P$ (réposition) $>\mathrm{N}$ (om).

Un gouverneur imperceptible : la prédication qui assigne le cas nominatif au sujet.

8. Cette idée est confirmée par presque tous les linguistes qui ont étudié l'arabe. Fassi Fehri (1993), par exemple, a remarqué que la direction du gouvernement est à droite du gouverneur en arabe, une idée répandue dans la grammaire traditionnelle. Cantarino (1975: 48): " the adjective functioning as an attributive apposition always follows its governing noun and is usually immediately after it ». Sibawayhi : " le gouverneur précède son gouverné en tout temps ».

9. En faisant nos recherches bibliographiques dans les ouvrages récents sur les adjectifs en arabe, nous avons constaté que presque rien n'a été fait dans ce domaine, mais nous avons trouvé de rares remarques qui s'accordent sur un seul point : l'arabe est un exemple de langue où les adjectifs sont toujours postnominaux. Laezlinger (2000) par exemple considère que l'arabe place les adjectifs attributs après le nom, comme c'est le cas du thaïlandais et de l'irlandais.

10. Wright, 1898 : II, 283.

11. Sur la question de considérer cet élément comme un pronom lié et non comme un marqueur d'accord, voir Fassi Fehri (1993 : chapitre 3).

12. Selon Milner (1989), cette notion traditionnelle dénote une propriété objective dans les langues naturelles et reflète une intuition fondée à propos du fonctionnement du langage.

13. "Substantives in apposition, or substantival apposition, refers (sic) to the asyndetical joining of one's substantive to another, both with the same syntactical function within the sentence. The purpose of this apposition is to identify or explain a given noun". (Cantarino: 1975) 
14. Voir pour une discussion de cette idée sur des bases cognitives Boudelaa \& Gareth (2002).

15. L'article en arabe prend deux formes: une forme proclitique (l'article défini) et une forme enclitique (l'article indéfini). La forme proclitique est assimilée à certaines consonnes initiales de $\mathrm{N}$ définies dans une liste close, pour des raisons phonologiques pures. La forme enclitique est souvent appelée "nounation» et constitue un trait caractéristique des langues sémites. (Kramsky, 1972 : 147-148).

16. Bouchard considère que les clitiques $l e$, la, les en français sont des lone DET (des déterminants isolés). Cette possibilité est offerte à cette langue parce que le Det porte le $\mathrm{Nb}$ et peut donc apparaître sans le N. En arabe, les clitiques sont des éléments pronominaux qui n'ont aucun rapport avec la classe des déterminants, mais qui entretiennent une relation étroite avec les paradigmes de l'accord (Jebali 2005).

17. La falsification consiste à soumettre de nouvelles propositions d'observations qui falsifient d'autres propositions d'observation (voir entre autres Lakatos, 1970).

18. L'article défini peut être employé comme un pronom relatif, mais c'est un emploi plutôt rare qui tend à disparaître de la langue (Wright, V.1, $1896: 270$ ).

\section{RÉSUMÉS}

La modification adjectivale en arabe standard pose plusieurs problèmes à la grammaire adaptative, qui constitue un modèle théorique minimaliste universaliste. Le minimalisme de ce modèle se base sur les propriétés des interfaces SM et CI pour expliquer les phénomènes langagiers, en particulier la modification adjectivale en français et en anglais. Nous démontrons que ce modèle ne peut fournir d'explication aux propriétés de la modification adjectivale en arabe. Pour ce faire, nous présentons les propriétés syntaxiques de la modification adjectivale dans cette langue et essayons d'appliquer le modèle adaptatif sur ces données.

The adjectival modification in standard Arabic poses several problems to the adaptative grammar, which constitutes a theoretical minimalist model. The minimalism of this model is based on the properties of the interfaces SM and CI to explain the linguistic phenomena, in particular the adjectival modification in English and French. We show that this model cannot provide explanation to the properties of the adjectival modification in Arabic. To reach our purpose, we will present the syntactic properties of the adjectival modification in that language, we will, then, try to apply the adptative model to explain these phenomena.

\section{INDEX}

Mots-clés : modification, adjectif, arabe, grammaire adaptative

\section{AUTEUR}

\section{ADEL JEBAL}

Université du Québec à Montréal 